\title{
Influence de la nutrition du hêtre (Fagus sylvatica L.) sur la sensibilité au chancre provoqué par Nectria ditissima Tul.
}

\author{
R. PERRIN et J. GARBAYE * \\ I.N.R.A., Station de Recherches sur la Flore pathogène dans le sol \\ Centre de Recherches de Dijon, 17, rue Sully, F 21034 Dijon Cedex \\ * I.N.R.A., Station de Recherches sur les Sols forestiers et la Fertilisation \\ Centre de Recherches forestières de Nancy, Champenoux, F 54280 Seichamps
}

\begin{abstract}
Résumé
Des plants de hêtres sont élevés sur tourbe fertilisée, dans laquelle on fait varier la disposition en éléments $\mathrm{N}, \mathrm{P}, \mathrm{K}, \mathrm{Ca}, \mathrm{Mg}, \mathrm{S}$. Après 8 à 9 mois d'élevage, les hêtres sont inoculés artificiellement avec Nectria ditissima, agent du chancre du hêtre. Une mesure mensuelle du développement longitudinal de la nécrose permet de suivre sa progression avec le temps. La nécrose s'étend rapidement de novembre à mars (phase d'invasion) puis régresse lorsque le phénomène de cicatrisation intervient (phase de résistance active). La variation du régime nutritif induit une forte variabilité de la taille des plants, mais l'extension de la nécrose est indépendante du diamètre du plant.

Un excès ou une carence en azote ou en phosphore accroissent nettement la sensibilité de l'ćcorce, tandis que les variations de l'alimentation potassique restent sans effet. La relation entre la gravité de la nécrose (appréciée par son développement longitudinal) et la teneur des différents éléments dans l'écorce met en évidence l'importance du calcium et du magnésium. La sensibilité de l'écorce est d'autant plus grande que la teneur en calcium ou en magnésium est plus faible. Cette étude révèle que la nutrition exerce une grande influence sur la sensibilité du hêtre au chancre et montre l'avantage liré d'une nutrition équilibréc sur la sensibilité du hêtrc à un agent d'altération de son écorce.

Enfin l'influence de la nutrition apporte une explication aux variations de l'état sanitaire des régénérations naturelles selon les conditions stationnelles, en particulier en hêtraic calcicole.
\end{abstract}

\section{Introduction}

Jusqu'à une période récente, la fertilisation forestière étail cantonnće à son usage en pépinière; elle est depuis une vingtaine d'années, pratiquéc en forêt où elle constituc un événement susceptible de modifier considérablement et brutalement la réceptivité des plantes aux parasites. En horticulture ou en agronomie où leur utilisation est ancienne, l'effet des fertilisants est mieux connu. Un excès d'azote (Stahel, 1946) ou de potasse (Moore \& BennetT, 1952) favorise la formation des chancres chez le pommier. En règle générale (HARE, 1966) de fortes doses d'azote accroissent la sensibilité des plantes aux parasites obligatoires et facultatifs, 
et de hauts niveaux de potassium et de phosphore tendent au contraire à l'abaisser. Mais cette règle souffre de nombreuses exceptions.

En ce domaine, les études forestières sont aussi abondantes que contradictoires. Dans certains cas, le travail du sol et la fertilisation améliorent la résistance écologique. Les dommages causés au pin sylvestre par le Lophodermium pinastri sont diminués de moitié dans les placeaux fertilisés avec N, P, K (ZotTL \& JUNG, 1964); un apport de $\mathrm{N}, \mathrm{P}, \mathrm{K}, \mathrm{Ca}, \mathrm{Mg}$ diminue notablement la nécrose de l'écorce du peuplier due à Chondroplea populea (Breuel, 1969). Dans d'autres cas la fertilisation est source d'aggravation de l'état sanitaire. Le châtaignier se montre plus sensible à l'Endothia parasitica à la suite d'application de fertilisants phosphorés et potassiques et Pinus elliotii manifeste une sensibilité accrue au Cronartium ribicola consécutive à un apport d'engrais $\mathrm{N}, \mathrm{P}, \mathrm{K}$ dont les conséquences fâcheuses dépassent largement le gain de croissance de l'arbre (HESTERBERG \& JURGENSEN, 1972).

Les résultats sont difficilement comparables compte tenu des nombreuses variations dans les caractères édaphiques, climatiques et dans la nature du matériel végétal. Ils ne permettent pas d'apprécier le véritable rôle des éléments majeurs à l'égard d'une maladie. II apparaît cependant qu'une nutrition déséquilibrée, qu'elle soit excessive ou insuffisante, a fréquemment pour la plante des conséquences fâcheuses plus ou moins marquées selon les éléments en cause.

La plante hôte possède un potentiel défensif qui mobilise une résistance biochimique au niveau cellulaire. La mise en cuvre et l'intensité de ce potentiel dépendent en grande partie de la nutrition. La compréhension de ces phénomènes passe nécessairement par une approche expérimentale. Chez le hêtre une des maladies les plus dommageables est due à Nectria ditissima qui produit une altération de l'écorce limitée par des bourrelets cicatriciels aboutissant à un faciès de chancre (Perrin, 1974). Cette maladie affecte principalement les régénérations naturelles. Sa gravité dépend des conditions stationnelles et peut atteindre, par place, la presque totalité des tiges au stade fourré (PERRIN \& VERNIER, 1979). Le chancre du hêtre hypothèque gravement la qualité potentielle des hêtraies dès leur plus jeune âge. La gravité de cette maladie varie de manière considérable avec les conditions stationnelles. VerniEr (1979) montre que la nutrition apparaît comme un des facteurs stationnels qui contribue le plus à cette variation, remarquant que le chancre est rare dans les stations calcicoles.

Disposant d'une méthode d'inoculation artificielle fiable et reproductible, nous nous proposons d'étudier expérimentalement la sensibilité du hêtre au chancre en fonction des conditions de nutrition.

\section{Matériel et méthodes}

\subsection{Matériel végétal}

Des faines d'une même provenance (forêt de Souilly, Meuse, sujette à de fortes attaques naturelles) ont été semées aux printemps 1977 et 1978 sur tourbe fertilisée en pots plastiques $\left(\begin{array}{ll}17 & 1\end{array}\right)$ afin d'obtenir au moins 4 plants par récipient. Chaque traitement comprend 20 plants répartis en 5 pots. 


\subsection{Modalités et dispositif expérimental}

Une première expérience conduite sur 2 ans (1977-1978) comporte 13 traitements, variantes d'une formule de fertilisation appelée dose 1 (LE TACON, 1974), soit pour $1 \mathrm{~m}^{3}$ de tourbe :

$75 \mathrm{~g}$ de $\mathrm{N}(226 \mathrm{~g}$ d'ammonitrate à 30 p. 100),

$75 \mathrm{~g}$ de $\mathrm{P}_{2} \mathrm{O}_{5}$ (167 g superphosphate triple à 45 p. 100),

$75 \mathrm{~g}$ de $\mathrm{K}_{2} \mathrm{O}$ (150 $\mathrm{g}$ de sulfate de potasse à $\left.50 \mathrm{p} .100\right)$,

$1 \mathrm{~kg}$ de calcaire magnésien (18-22 p. $100 \mathrm{MgO})$,

$80 \mathrm{~g}$ d'un mélange d'oligoéléments.

La variation séparée des éléments $\mathrm{K}$ et $\mathrm{S}$ d'une part et $\mathrm{Ca}$ et $\mathrm{Mg}$ d'autre part est obtenue à partir de chlorures de ces éléments.

A partir de cette dose de base (dose 1) constituant le témoin $\mathrm{T}$, un des éléments a été soit supprimé totalement $(-\mathrm{N},-\mathrm{P},-\mathrm{K},-\mathrm{Ca},-\mathrm{Mg},-\mathrm{S})$ soit sa quantité a été doublée $(2 \mathrm{~N}, 2 \mathrm{P}, 2 \mathrm{~K}, 2 \mathrm{Ca}, 2 \mathrm{Mg}, 2 \mathrm{~S})$, les autres éléments demeurant inchangés.

Une seconde expérience réalisée en 1978-1979 fait appel à une fertilisation de base équivalente à 2 fois la dose 1 , et fait intervenir une variation plus graduelle pour les éléments $\mathrm{N}, \mathrm{P}, \mathrm{K}$. Pour chacun de ces éléments on réalise 4 traitements caractérisés par une quantité définie relativement à la dose $1(\times 4, \times 1, \times 1 / 2, \times 1 / 4)$; les autres éléments étant apportés selon la fertilisation de base. Aux 12 modalités ainsi obtenues s'ajoute le traitement T, ou les éléments sont apportés selon la fertilisation de base. Au terme de cette expérience l'analyse chimique de l'écorce (non nécrosée) a été effectuée (teneurs totales en azote, phosphore, potassium, calcium et magnésium).

\subsection{Inoculation artificielle}

Expression et mesure de la gravité de la maladie

Au terme d'une saison de végétation les hêtres sont inoculés dès que le feuillage marcescent perd sa coloration verte, et après une période d'alimentation hydrique réduite, conditions optimales pour l'initiation du chancre à Nectria ditissima (VERNIER, 1978).

\subsection{Méthode d'inoculation artificielle}

Après désinfection superficielle de l'écorce à l'alcool à $90^{\circ}$, une blessure est réalisée à l'aide d'une lancette de Bensaude à l'entre-nœud immédiatement supérieur aux traces cotylédonaires. Le traumatisme produit est ainsi parfaitement contrôlé ei reproductible. En fixant la taille de la blessure (longueur et profondeur) on diminue considérablement la variation induite par les différences de réalisation de cette blessure. L'inoculum est apporté sous forme mycélienne (portion de culture de 
$N$. ditissima sur malt agar prélevée à l'emporte-pièce $9 \mathrm{~mm}$ au front de croissance d'une souche isolée en forêt de Souilly, Meuse) placé au contact de la partie lésée. Pour l'entretien d'une ambiance humide favorable au développement du parasite, on superpose à l'inoculum un coton imbibé d'eau stérile. Le tout est maintenu et protégé par une bande de parafilm.

Une inoculation est réalisée en novembre 1977 et 1978 sur chaque plant d'un traitement. Cinq d'entre eux subissent en plus une blessure témoin (absence de champignon).

Après 6 à 8 jours entre 10 et $20^{\circ} \mathrm{C}$, on retire l'ensemble protection et inoculum. Les plants sont maintenus en serre hors gel pendant l'hiver, conditions favorables à l'initiation de la nécrose, puis sont transférés en pépinière début mars.

\subsection{Acquisition des données}

Chaque plant donne lieu à une mesure de hauteur totale, et du diamètre de sa tige au point d'inoculation, le jour même de l'inoculation. L'évolution ultérieure de la nécrose corticale est appréciée par la mesure mensuelle de son développement dans le sens vertical ( $L$ ) et horizontal ( $\mathrm{P}=$ portion de circonférence) pendant une saison de végétation. En forêt, le chancre évolue chaque année selon un cycle annuei débutant par une extension pendant le repos végétatif, interrompue par la cicatrisation pendant la saison de végétation. Le chancre s'étend sous forme de rides concentriques correspondant à la répétition d'une évolution annuelle. On admet que l'influence de la nutrition puisse s'exercer de la même manière au cours de chaque évolution annuelle.

La comparaison des différents traitements fait appel à l'analyse de variance à 1 ou 2 facteurs contrôlés, à la méthode du 1 corrigé de Bonferoni $\left(1^{\mathrm{r}}\right.$ expérience) ou le test $U$ de Mann et Whitney (2 expérience).

\section{Résultats}

La nutrition peut exercer une double influence sur la sensibilité du hêtre au chancre. Elle peut, d'une part modifier directement la réceptivité propre de l'écorce, et d'autre part agir indirectement par la taille et la vigueur du plant.

\section{TABleaU 1}

Evolution du chancre avec le temps (tous traitements confondus).

Canker spreading with time (all treatments gathered).

\begin{tabular}{l|c|c|c|c|c|c}
\hline & $12 / 77$ & $1 / 78$ & $2 / 78$ & $3 / 78$ & $4 / 78$ & $5 / 78$ \\
\cline { 2 - 3 } & $\mathrm{L}_{1}$ & $\mathrm{~L}_{2}$ & $\mathrm{~L}_{3}$ & $\mathrm{~L}_{4}$ & $\mathrm{~L}_{5}$ & $\mathrm{~L}_{6}$ \\
\hline Date de mesure $\ldots \ldots \ldots \ldots$ & 3,68 & 4,18 & 4,64 & 5,07 & 4,87 & 4,52 \\
\hline
\end{tabular}




\subsection{Extension du chancre au cours du temps}

Les 2 critères choisis ( $\mathrm{L}$, ou $\mathrm{P}$ ) décrivent l'extension du chancre de manière très semblable. Les résultats présentés dans le tableau 1 sont exprimés à l'aide de la variable $\mathrm{L}$ qui présente la plus forte amplitude, reflétant le développement préférentiel du chancre dans le sens de la tige. tinctes :

L'évolution temporelle du chancre se réalise selon 2 phases successives dis-

- une rapide extension du champignon dans les tissus de l'hôte alors en repos végétatif $\left(\mathrm{L}_{1}\right.$ à $\left.\mathrm{L}_{1}\right)$. L'absence de réaction active de l'hôte à l'invasion des tissus corticaux caractérise cette phase dite phase d'invasion;

- un arrêt, puis une régression de la nécrose marquant une réaction active de l'hôte en coïncidence avec le débourrement végétatif des plants (échelonné entre le $2 / 78$ et $4 / 78$ ). C'est la phase dite de résistance active.

\subsection{Influence du diamètre du plant sur l'extension du chancre}

Quarante-deux plants supplémentaires élevés sur tourbe fertilisée (traitement témoin complet) recouvrant une large gamme de diamètre au collet (2 à $6,5 \mathrm{~mm}$ ) ont été inoculés.

Les résultats rassemblés dans le tableau 2 indiquent que l'extension longitudinale de la nécrose est indépendante du diamètre du plant.

\section{TABLeau 2}

Régression entre le diamètre du plant (au point d'inoculation) et le développenentent vertical (L) du chancre ( $\mathrm{mm}$ ). Evolution temporelle (inoculation 11/77).

Regression botweon seedlings diameter (inoculation site) and vertical elongation ( $L$ - mm) of canker. Time evolution (date of inoculation 11/77).

\begin{tabular}{|c|c|c|c|c|c|c|c|}
\hline & $\begin{array}{l}\text { Diamètre } \\
(\mathrm{mm})\end{array}$ & & & & & & \\
\hline Date $d z$ mesure & & $\begin{array}{c}12 / 77 \\
\mathrm{~L}_{1}\end{array}$ & $\begin{array}{c}1 / 78 \\
\mathrm{~L}_{2}\end{array}$ & $\begin{array}{c}2 / 78 \\
L_{3}\end{array}$ & $\begin{array}{c}3 / 78 \\
L_{1}\end{array}$ & $\begin{array}{c}4 / 78 \\
\mathrm{~L}_{j}\end{array}$ & $\begin{array}{c}5 / 78 \\
\mathrm{~L}_{i}\end{array}$ \\
\hline Moyenne ... & 3.93 & 4,4 & 4,9 & 6,3 & 8.5 & 80 & 5.9 \\
\hline Ecart-type ..... & 1.25 & 1,2 & 2.1 & 2.5 & 4,2 & 425 & 1,9 \\
\hline $\begin{array}{l}\text { Coefficient de régres- } \\
\quad \text { sion } \ldots \ldots \ldots \ldots \ldots\end{array}$ & & -0.08 & $-0,14$ & $-0,20$ & -023 & -023 & $-0,36^{*}$ \\
\hline
\end{tabular}

: Significatif au seuil de 5 p. 100. 
Toutefois la taille du plan interfère sur la sensibilité du hêtre au chancre en mai 1978 donc uniquement en phase de cicatrisation. La cicatrisation s'opère avec difficulté sur les plants de faible diamètre. L'extension de la nécrose dépend essentiellement de la sensibilité de l'écorce au cours des 5 premiers mois.

\subsection{Influence de la nutrition sur l'extension du chancre (1 $1^{\text {re }}$ expérience)}

La comparaison de l'influence des différents éléments peut être faite à partir de l'évolution du chancre retracée à l'aide de diagrammes (fig. 1).

La hiérarchie établie au cours des premiers mois connaît peu de bouleversements par la suite. Au terme de la phase d'invasion 2 traitements se distinguent des autres. Les hêtres privés de phosphore et d'azote montrent une sensibilité accrue à $N$. ditissima. Il en est de même, dans une moindre mesure, des hêtres développés sur un milicu dépourvu de magnésium. Le défaut de phosphore entraîne une augmentation de la sensibilité de l'écorce qui se manifeste dès le début de l'évolution de la nécrose.

Au cours de la phase de résistance active la réduction de la taille de la nécrose connaît de substantielles variations. La cicatrisation s'opère très difficilement lorsqu'en particulier le magnésium, le phosphore ou l'azote font défaut. Par contre la cicatrisation est très active chez les hêtres ayant bénéficié d'une nutrition enrichie en calcium et en azote. Malgré les différences très marquées, seuls les traitements $2 \mathrm{Ca}$ et $2 \mathrm{~N}$ sont significativement différents du traitement - $\mathrm{Mg}$ en raison d'une forte variabilité. Celle-ci exprime le fait que la cicatrisation s'est effectuée préférentiellement sur certains plants oblitérant totalement le chancre, alors qu'elle reste sans effet sur les autres.

\subsection{Influence des différents éléments (2' expérience)}

L'expérience faisant intervenir une variation graduelle des éléments majeurs permet d'en préciser l'influence propre. Les résultats sont présentés à la date où les différences sont les plus marquées, et intègrent la phase d'invasion et le début de la phase de résistance active.

\subsection{Influence de la nutrition azotée}

Les résultats qui font l'objet du diagramme de la figure 2 indiquent qu'un excès ou une carence en azote par rapport à la fertilisation de base accroissent la sensibilité du hêtre au chancre. C'est toutefois l'excès d'azote qui a l'effet le plus prononcé.

\subsection{Influence de la nutrition phosphorée}

Le diagramme de la figure 3 établi à partir des mesures effectuées en juin 1979 révèle qu'une carence ou plus encore un excès en phosphore favorisent le développement du chancre. 


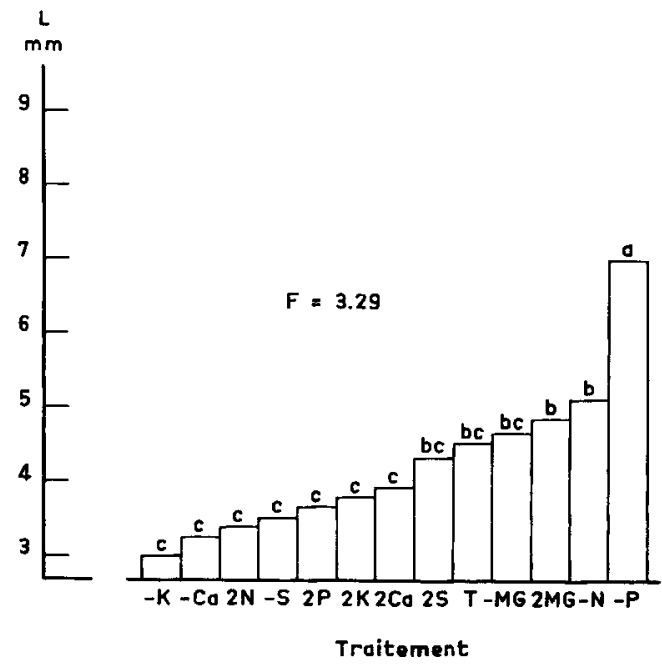

JANVIER 1978

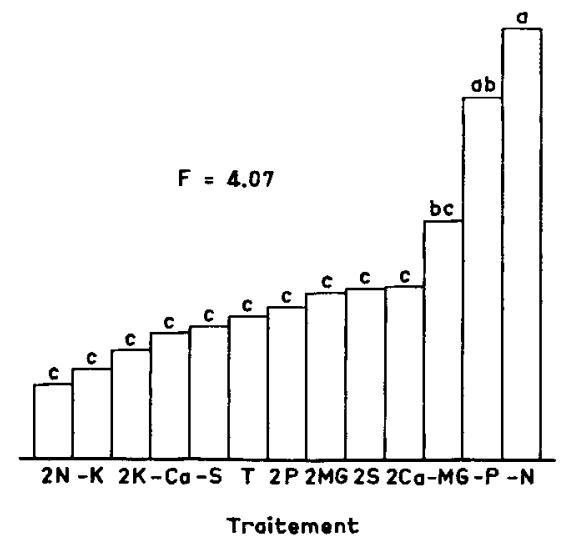

MARS 1978

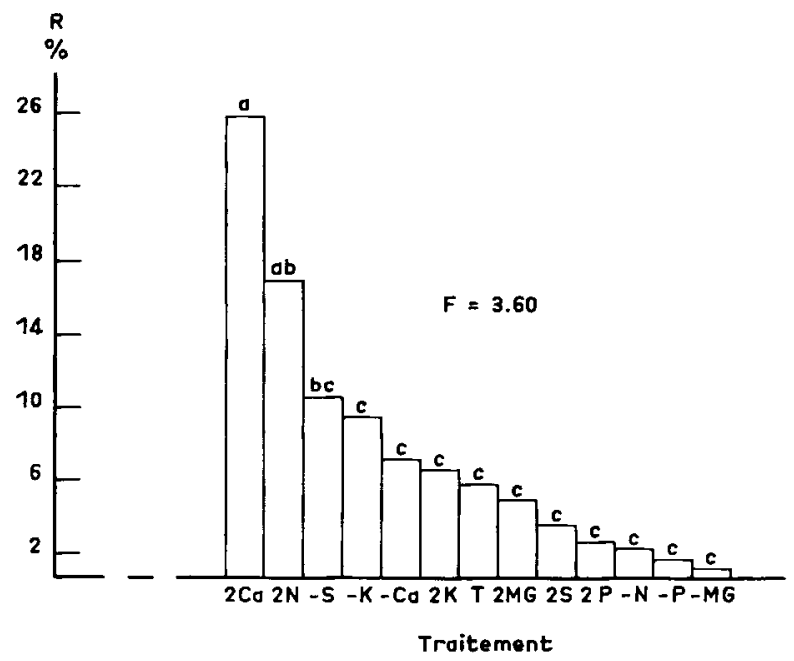

\section{REDUCTION DE LA NECROSE

ENTRE AVRIL ET JUIN 78

FIG. 1

Influence de la nutrition sur la longueur du chancre.

Nutrition's influence on canker vertical development.

Les traitements indicés d'une même lettre ne sont pas significativement différents au seuil de 5 p. 100 - t corrigé Bonferoni.

Treatments with the same letter were not statistically different at the $5 \mathrm{p}$. 100 level using Bonferoni test. 


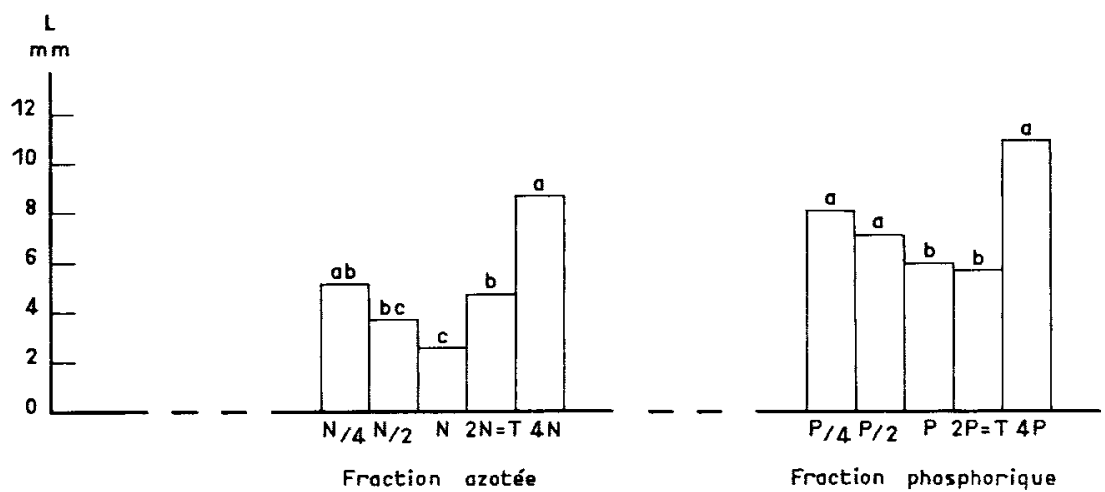

FIG. 2

Longuteur de la nécrose (mai 1979 - 5 mois d'évolution) en fonction de la fertilisation azotée.

Effect of nitrogen fertilization on vertical development of the necrose (may $1979-5$ months evolution period).

Les traitements indicés d'une même lettre ne sont pas significativement différents au seuil de 5 p. 100. Test U Mann et Whitney.

Treatments with the same letter were not statistically different at the $5 \mathrm{p} .100$ level using $U$ test Mann and Whitney.

FIG. 3

Longueur de la nécrose induite artifiellement sur des hîtres en fonction de la fertilisation phosphorée (juin 1979).

Effect of phosphorus fertilization on vertical extent of the necrose artificially induced on beech seedlings (June 1979).

Les traitements indicés d'une même lettre ne sont pas significativement différents au seuil de 5 p. 100. Test U Mann et Whitney.

Treatments with the same letter were not statistically different at the $5 \mathrm{p}$. 100 level using $U$ test Mann and Whitney.

\subsection{Influence de la nutrition potassique (fig. 4)}

Il n'est jamais possible de distinguer une différence significative de sensibilité chez les hêtres ayant bénéficié de fertilisations potassiques variées. Toutefois la nécrose a tendance à être plus étendue en situation de carence. 


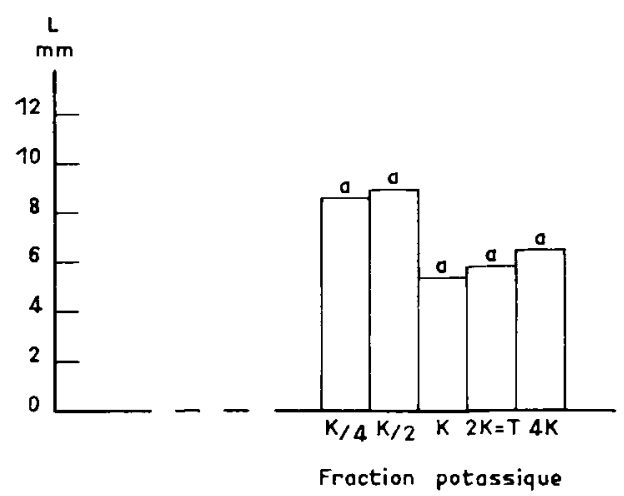

FIG. 4

Longueur moyenne de la nécrose en fonction de la fertilisation potassique (juin 1979).

Effect of potassium fertilization of vertical extent of the necrose induced by $\mathrm{N}$. ditissina (June 1979).

Les traitements indicés d'une même lettre ne sont pas significativement différents au seuil de 5 p. 100. Test $U$ de Mann and Whitney.

Treatments with the same letter were not statistically different at the $5 \mathrm{p}$. 100 level using $U$ test $M$ amn and Whitney.

\subsection{Relation entre la gravité de la maladie et la teneur des éléments dans l'écorce}

L'examen des variations de la taille de la nécrose en fonction de la tencur d'un élément dans l'écorce montre :

- une étroite liaison avec la nutrition calcique et magnésicnne. Le coefficient de corrélation linéaire entre la longueur du chancre (mai 1979) et la teneur de l'écorce en calcium et magnésium est très élevé $--0,85$ et $-0,78$ respectivement (fig. 5) - Plus l'écorce est riche en ces éléments, moins la nécrose s'étend. De plus on améliore considérablement le coefficient de corrélation dans le cas du calcium $(0,947)$ en éliminant les traitements associés à la variation de l'élément $\mathrm{K}$ (antagonisme $\mathrm{K} / \mathrm{Ca}$ ). Par la variation de la fertilisation en éléments majeurs $\mathrm{N}, \mathrm{P}, \mathrm{K}$, on induit des modifications dans la nutrition calcique qui retentissent sur la sensibilité du hêtre au chancre bien que l'expérience précédente n'ait pas abouti à unc différence significative entre $-\mathrm{Ca}$ et $2 \mathrm{Ca}$;

- la relation précédente ne s'applique pas aux plants du traitement complet et équilibré, en particulier dans la relation entre la gravité de la nécrose et la teneur en calcium de l'écorce (fig. 5 a). Malgré une teneur réduite, la nécrose observée est peu développée. Ce résultat traduit l'importance de l'équilibre dans la nutrition des plantes et leur sensibilité aux parasites corticaux;

— aucune liaison évidente n'apparaît avec les autres éléments étudiés. 

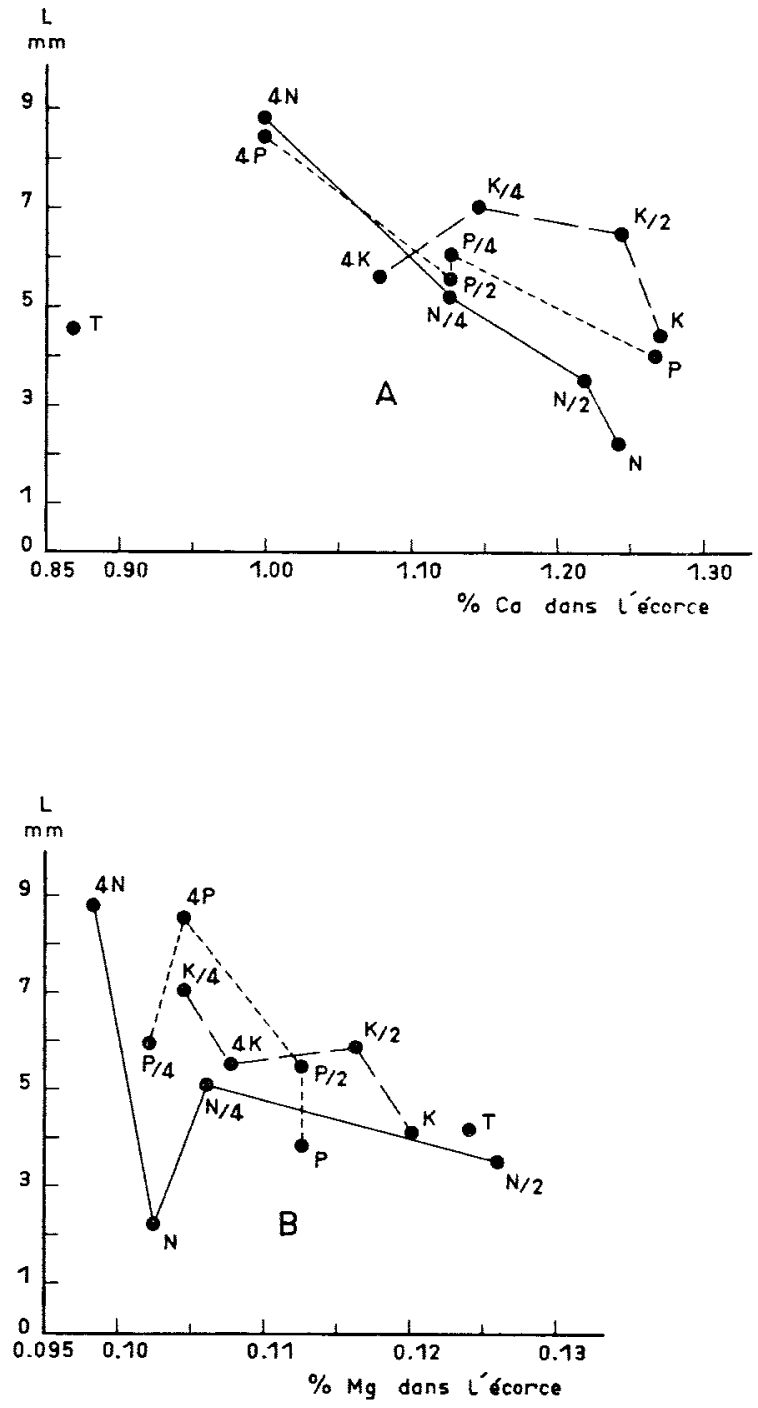

FIG. 5

Relation entre la gravité de la nécrose et la teneur de l'écorce en calcium (A) et en magnésium (B).

Relationship between disease severity and calcium $(A)$ and magnesium $(B)$ bark contents. 


\section{Discussion, conclusion}

Cette étude a révélé que la nutrition exerce une grande influence sur la sensibilité du hêtre au chancre dû à Nectria ditissima. L'azote et le phosphore sont les 2 éléments qui agissent le plus nettement sur la sensibilité de l'écorce, qui paraît indifférente aux variations d'alimentation potassique. La sensibilité de l'écorce est accrue aussi bien dans le cas d'un excès que d'une carence de ces 2 éléments. Deux autres éléments $\mathrm{Ca}$ et $\mathrm{Mg}$, affectent considérablement la sensibilité du hêtrè au chancre. Celle-ci est d'autant plus élevée que la teneur de l'écorce en calcium et en magnésium est plus basse. L'activité pathogène du $N$. ditissima provoque des altérations du métabolisme de l'hôte aboutissant à des dépenses supplémentaires d'énergie. A ce titre la disponibilité en phosphore et à un niveau moindre en calcium (activateur des phosphatases) intervient de façon fondamentale dans la faculté de l'hôte à s'opposer au parasite. Au cours de la phase de résistance active, l'hôte doit accomplir une synthèse protéique accrue nécessitant un surcrô̂t d'énergie et une fourniture supplémentaire d'azote et de magnésium cofacteur de nombreuses réactions enzymatiques.

La variation de la gravité de la maladie en forêt (Perrin \& Vernier, 1979) s'accorde avec ces résultats, bien qu'en forêt des excès d'alimentation azotée ne puissent atteindre les proportions de l'expérience. De même les variations de disponibilité en phosphore n'ont pas un rentissement très marqué sur la gravité de la maladie. Dans ce dernier cas c'est sans doute la mycorhization qui atténue sensiblement l'influence de l'élément $\mathbf{P}$ sur la gravité de la maladie en forêt, alors que les racines des hêtres soumis à l'expérience sont pas ou très peu mycorhizées.

Mais la coïncidence entre l'état sanitaire des régénérations naturelles ct l'influence de la nutrition est particulièrement flagrante vis-à-vis du calcium.

Les stations où la proportion de tiges atteintes est la plus faible correspondent aux sols les plus calcaires. Vigouroux (1981) constate également que la bactériose du pêcher est plus grave sur sols calcaires. Cette liaison suggère l'intervention de l'ion $\mathrm{Ca}^{2}+$ dans la réalisation de liaisons particulières avec les composés pectiques, en formant notamment des pectates réputés moins accessibles aux activités enzymatiques des champignons. La liaison entre la sensibilité d'un tissu végétal à un agent pathogène et sa teneur en $\mathrm{Ca}^{2}+$ a été rapportée à de nombreuses reprises dans le cas de maladies très différentes (SKOU, 1979).

Le bénéfice tiré d'une nutrition équilibrée apparaît nettement dans cette étude et confirme le caractère optimal de la fertilisation proposée par Delran, Garbaye \& LE TACON (1975). Outre l'avantage considérable obtenu sur la croissance des plants, elle minimise la sensibilité du hêtre à l'agent du chancre Nectria ditissima.

\section{Summary \\ Influence of the nutrition on the Nectria ditissima canker sensibility of beech (Fagus sylvatica L.)}

Beech seedlings have been raised on fertilized light peat with variations in $N, P, K$, $\mathrm{Ca}, \mathrm{Mg}$ and $\mathrm{S}$ supplies. Eight to 9 months old beeches have been wound inoculated with Nectria ditissima, cause of beech canker. Time evolution of the necrosis has been followed 
by a monthly assessment of it's vertical extent. The necrose spread rapidly between November and March (invading period) then decreases as soon as healing over occurs (active resistance period). Variations in nutritive supplies induce a high variability in seedlings size, but no relation can be found between necrose's extent and seedling diameter.

$\mathbf{N}$ or $\mathbf{P}$ excess or deficiency increase bark sensibility, whereas $\mathrm{K}$ variations do not affect it. The relationship between the necrose severity (expressed by it's vertical extent) and the mineral contents of the bark reveals the importance of calcium and magnesium. Bark sensibility is more especially higher as it's calcium and magnesium contents are lower. The nutrition has a great influence on the canker sensibility of beech. Moreover a well balanced fertilization reduces the bark sensibility.

Variation in disease severity with stand conditions in forest can receive a good explanation because of the nutrition influence, specially in calcarcous beech stands.

Reçu le 20 septembre 1983.

Accepté le 11 janvier 1984.

\section{Références bibliographiques}

Bruel K., 1969. Uber den Einfluß edaphisher Faktorum auf die Prädisposition einer Papelplautage gegenüber Dothichiza populea Sacc. et Br. Arch. Forstwess, 18, 1265-1272.

Delran S., Garbaye J., Le Tacon F., 1975. Production rapide de plants feuillus sur tourbe fertilisée. Nouveaux résultats. R.F.F., 6, 436-448.

Hare R.S., 1966. Physiology of resistance to fungal discases in plants. Bot. Rev., 32, 2, 95-137.

Hesterberg G.A., Jurgilinsen M.F., 1972. The relation of forest fertilization to discase incidence. The Forestry Chronicle, April, 92-95.

LE TACON F., 1974. Recherches des meilleures conditions de production de plants de hêtre. Premiers résultats. R.F.F., 4, 299-305.

Moore M., BennetT T.M., 1952. Scab, canker and branch blister of apple at East Malling in relation to nutritionnal treatments of the host. Ann. Appl. Biol., 39, 588-598.

Perrin R., 1974. Le chancre du hêtre. R.F.F., XXVI, 3, 219-221.

Perrin R., Vernier F., 1979. Le chancre du hêtre : influence des conditions stationnelles sur la gravité de la maladie. R.F.F., XXIX, 4, 286-297.

Skou J.P., 1979. Radiation induced sprout and growth inhibition in vegetables with special reference to the susceptibility to microbial attacks and the effect of calcium. R.I.S.O. National Laboratory, 305 p.

Stahel M., 1946. Die Krebskrankheit unserer Obstbaüme ihre Ursachen und Bekämpfung. Schweiz. Z. Obst. U. Weinh., 55, 285-291 (Hort. Abstract., 16, 1946, 1899).

VERNIER F., 1979. Etude de l'influence de quelques facteurs sur la sensibilité du hêtre au chancre. Mémoire de fin d'études E.N.I.T.E.F., 68 p.

Vigouroux A., 1981. Influence du sol sur la sensibilité du pêcher au dépérissement bactérien. Compte rendu des séminaires du groupe d'étude des racines, 8, 202-203.

ZotTl A., Jung P., 1964. Ernährungszustand und Lophodermium Befall von Pinus silvestris. Naturwissenschaften, 51, 24, 643. 